Im Zweifel treu? Der schweigende Abraham

Brigitte Boothe

\title{
Die exorbitante Zumutung
}

Der göttliche Befehl an Abraham, den Sohn Isaak zu opfern (Abrahams Versuchung. Bestätigung der Verheissung, Genesis 22, 1-19), ist bestürzend. Das ist für den Leser oder Hörer der biblischen Erzählung von Abrahams Versuchung offensichtlich. Es versteht sich von selbst: Abraham hat allen Grund, am göttlichen Wohlwollen zu zweifeln. Eben darum wird die Geschichte erzählt: Weil alles dafür spricht, die exorbitante Zumutung zurückzuweisen (Boothe 2006). Offensichtlich muss Abraham an einem göttlichen Souverän zweifeln, der ihm Leben, Familie und Zukunft zerstört:

- Gott hat Abraham reiche Nachkommenschaft durch Isaak versprochen. Wie kann sich das Versprechen erfüllen, wenn Isaak stirbt, bevor er Gelegenheit hatte, Kinder zu zeugen?

-Wie kann Abraham sich durch den göttlichen Souverän begünstigt sehen, wenn dieser Abrahams Liebe zu seinem Sohn Isaak missachtet?

-Wie kann Abraham vor Sohn und Mutter rechtfertigen, dass er dem göttlichen Souverän gehorcht und die Tötung des Sohnes in Kauf nimmt und vollstreckt?

Die Antworten auf diese Fragen liegen auf der Hand:

- Wenn Gott die Opferung des Isaak befiehlt, dann wird sein Versprechen hinfällig. Der göttliche Ratschluss verfällt dem Zweifel.

- Wenn Gott mit seinem Befehl die Interessen von Vater und Sohn missachtet, ist er Feind, nicht Freund. Er steht unterVerdacht.

-Wenn Abraham sich zum Vollstrecker des göttlichen Befehls macht, verwirft er die eigene Väterlichkeit. Ein göttlicher Tyrann als destruktiver Imperator.

\section{Selbstbewusstsein und Mut zum Zweifeln}

Es scheint, mit Kant (1798), eindeutig: Die exorbitante Zumutung entlarvt sich als Anmassung, ist nicht göttlichen, sondern betrügerischen Ursprungs. Das aufgeklärt selbstbewusste Individuum, zu 
vernünftiger Skepsis und begründetem Zweifel fähig, schleudert sie weg.

"Zweifle an allem wenigstens einmal und wäre es auch der Satz ¿Zweimal zwei ist vier!)."So lautet einer der berühmten Aphorismen des Aufklärers Georg Christoph Lichtenbergs (1742-1799). Es ist die Maxime kritisch selbstbewusster Courage, die sich nicht beugen lässt. Fähigkeit zum aufrechten Gang, Skepsis, Zweifel, kritische Exploration, wissenschaftlicher Geist, so sieht auch Freud (1927, 377) das aufgeklärt selbstbewusste Individuum:

"die Stimme des Intellekts ist leise, aber sie ruht nicht, ehe sie sich Gehör geschafft hat. Am Ende, nach unzählig oft wiederholten Abweisungen, findet sie es doch. Dies ist einer der wenigen Punkte, in denen man für die Zukunft der Menschheit optimistisch sein darf ...

So appelliert Freud in seinem prominentesten religionskritischen Werk Die Zukunft einer Illusion (1927) an die Selbst- und Weltkenntnis liebes- und arbeitsfähiger Menschen der Moderne, das kostbare Gut kritikfähigen Selbstbewusstseins zu verteidigen. Die religiöse Orientierung bindet Energien, derer der Einzelne bedarf, um sein intellektuelles Potential optimal im Dienst der denkenden und handelnden Konfrontation mit der Realität einzusetzen. Das autonome Individuum würde

»nicht unter dem Druck eines religiösen Systems aufwachsen. Dadurch, dass er seine Erwartungen vom Jenseits abzieht und alle freigewordenen Kräfte auf das irdische Leben konzentriert, wird er wahrscheinlich erreichen können, dass das Leben für alle erträglich wird und die Kultur keinen mehr erdrückt." (Freud 1927, 373-374)

Die Bereitschaft, dem Kosmos die Gestalt des Göttlichen zu verleihen, hält die Psyche in tiefer Infantilität gefangen. Glauben ist nur möglich auf der Basis affirmationswilliger Borniertheit, denn religiöse "Lehrsätze verlangen [...] Glauben für ihre Inhalte, $[\ldots]$ ohne ihren Anspruch zu begründen" (Freud, 1927, S. 347). Sie wirken stattdessen als Heils-, Erlösungs-, Rettungs- oder Exkulpierungsversprechen, ohne jemals diese in Aussicht gestellten Prämien einzulösen. Die Religion schüchtert autoritär ein, tabuiert den Zweifel, verordnet Gläubigkeit; - Haltungen, die in einem demokratischen Staatswesen und für mündige Personen obsolet sind. Die Glaubensinhalte bieten Kompensation für die unstillbare Vater- und Elternsehnsucht und für die Unfähigkeit, den eigenen Tod als Auslöschung der eige- 
nen Existenz psychisch zu integrieren. Es gibt Ursachen für eine gläubige Haltung, aber keine guten Gründe für Glaubenssätze und Glaubensinhalte. Die Religion gibt sich mit dem Trösten zufrieden und verzichtet auf zweifelnd kritische Intelligenz zuungunsten der Vernunft, meint Mackie (1987, 415), ein zugespitztes Diktum des atheistischen Religionskritikers Robinson aufgreifend. Ist dies durchschaut, so führt kein Weg mehr zurück in den religiösen Infantilismus.

Der Mut zum Zweifel, die Vermehrung von Urteilsfähigkeit, von Selbst- und Weltkenntnis ist für den Moralisten und Realisten Freud ein hoher Wert. Das Individuum kann lernen, Täuschung und Selbsttäuschung, Abwehr und Illusionsbildung zu analysieren. Es kann lernen, Passivierung und Eskapismus zu überwinden. Es kann Gründe geben und Gründe verlangen; es kann und soll für sein Handeln einstehen. Es erwirbt dynamische Reflexivität, Beziehungsintelligenz, die Fähigkeit zur Analyse von Motiven und zu flexibler Perspektivenübernahme (Boothe 2006a).

DieWissenschaft geniesst auch ein Jahrhundert nach Freud Respekt, hat aber den Stein der Weisen nicht. Die Wissenschaftler erweisen sich nicht als Vorbilder für gelebte Vernunft. Dennoch: Freud (1928) empfiehlt den Humor als die Geisteshaltung, die der Fragilität der menschlichen Daseinsverfassung, die dennoch auf Vollendung und Erweiterung drängt, am besten gerecht wird. Wer den psychischen Verhältnissen auf der Spur ist, ist unterwegs zu den Möglichkeiten der Freiheit, die menschliche Existenzen sich schaffen können. Er kann zu einer Geisteshaltung gläubiger Kritiklosigkeit nicht mehr zurückfinden.

\section{Mutig ist Abraham, selbstbewusst ist er, und er schweigt}

Die Versuchungserzählung thematisiert aber gar nicht gläubige Kritiklosigkeit. Kants, Lichtenbergs und Freuds Empfehlung "Sei kritisch und übe dich in der Kunst des Zweifels« läuft hier ins Leere, denn die Erzählung provoziert gerade zweifelnden Protest. Die Figur des Abraham in der Versuchungserzählung ist gar nicht gehorsam, borniert, selbstverleugnend oder kritiklos. Längst ist Abraham narrativ positioniert als Mann, der mit Gott erfolgreich redet und rechtet, der klug argumentiert und konfrontiert, der weiss, was er will und im Diesseits das Seine sucht, ein trickreicher Geschäftsmann, klug, bisweilen skrupellos im Umgang mit Menschen. Der göttliche Souverän 
hat ihn, der damals Abram hiess, ausgewählt, um ihn, samt Frau, Gesinde und Besitz, zum Exodus aus der Heimat herauszufordern, Segen, Prosperität und imperiale Expansion verheissend. Abram bewährte sich mit Klugheit, List, Eloquenz, Verhandlungsgeschick, Mut, Ressourcen- und Machtbewusstsein in der Fremde, regulär unterstützt und ermutigt durch göttliche Verheissungen. Er erstarkte an Courage, Bewährungskompetenz und Selbstbewusstsein und stellt den göttlichen Souverän in Gen 15 hinsichtlich der Einlösung der Versprechen als kritisch Zweifelnder zur Rede. Gott gewährt dem wachen Geist seines Hoffnungsträgers und Favoriten explizit Anerkennung. Und dieser macht seinen Einfluss geltend. Er bewährt sich darüber hinaus als kluger und erfolgreicher Bündnispolitiker. Dies sind die Essentials der Ereignisse, die Gen 22 vorausgehen.

Als Gott den Abraham versucht, findet er in diesem eine profilierte welterfahrene Persönlichkeit, die Gott gegenüber nicht nur verehrungsbereit, sondern auch verhandlungs-, gesprächs- und streitfähig ist. Gott konfrontiert den Abraham als kraftvolle Persönlichkeit mit Intelligenz und Behauptungswillen. Abraham stellt sich der Aufgabe ohne Zögern und handelt mit eindrucksvoller Umsicht: Er zieht niemanden ins Vertrauen und in Mitleidenschaft, übernimmt also das Projekt als das seinige. Er gestaltet das Moratorium der Reise als ein Schweigender, macht aber auf die Frage des Kindes nach dem Opfertier explizit, daß er Gott als den Wählenden sieht und daß die Wahl etwas ist, das in der Schwebe sein könnte: Es ist Gott, der sich ein Schaf ersehen wird. Er hört auf das Gebot, inne zu halten, opfert den Widder, der in der Nähe erscheint, schweigt zum großen Segen und kehrt zu den Knechten zurück. Er beharrt nicht auf dem, was ihm durch göttliche Privilegierung zuteil geworden war, er regrediert nicht zum verantwortungsentlasteten Befehlsempfänger und übernimmt das Projekt in die eigene Planungs- und Handlungsregie. Er nimmt den Segen entgegen und wird weiter ein Mann der Tat und der Umsicht sein.

Warum schweigt Abraham ausgerechnet dort, wo beredter Positionsbezug erwartbar ist? Er bekundet Hier bin ich, unterlässt jeden Kommentar, gegen Gott, gegen die Menschen, und tut wie befohlen. Warum bleibt die Kommentierung des Beredten, Tüchtigen, Erfahrenen und Schlauen aus? Der Verzicht auf Worte angesichts einer Zumutung, die Worte des Widersprechens nahelegt, hat als spezifisches und wirkungsvolles Gestaltungsmerkmal dieser Erzählung $\mathrm{zu}$ gelten. Indem Abraham schweigend tut wie geheissen, verzichtet er auf die Bekundung von Zweifel, Protest und Eigenwillen. Was er denkt, bleibt verborgen. Am Ende des Reisemoratoriums nimmt 
Gott die Forderung, den Sohn zu opfern, zurück. Er beutet den Dienenden nicht aus.

Abraham befindet sich zum Zeitpunkt der Loyalitätsprobe im Zustand der Fülle und unterliegt daher, was die verantwortungsbewusste Pflege seiner materiellen und lebendigen Güter angeht und was die Ehrfurcht vor dem Herrn betrifft, durchaus im Risiko, die Abhängigkeit von der privilegierenden souveränen Instanz zu verkennen und die erworbenen Ressourcen in eigener Sache, bei gelockerter Loyalität zu nutzen und zu mehren. Nicht, dass Abraham dies bereits hätte erkennen lassen. Gott aber hat ihn umgekehrt bereits zum Vertrauten und Gefährten werden lassen und ihn auch in prekären Situationen rückhaltlos unterstützt, beispielsweise, als Abraham sich durch Prostitution der eigenen Gattin, ausgegeben als Schwester, auf sündigem Weg Sicherheiten und materielle Vorteile erschlich, auch als er auf Sarahs Wunsch den eigenen Sohn Ismail in die Wüste schickte. Auf dem Höhepunkt seiner Erfolge könnte Abraham gottvergessen werden.

Er wird es aber nicht. Im schweigenden Opfergang anerkennt er den Anspruch des Objekts, an das er sich bindet. Er erfüllt den Anspruch im Dienst am Objekt. Dienst ist Engagement im Interesse des Objekts, um seinetwillen. Abrahams Ausdruck des Gebundenseins ist die Bereitschaft, im Dienen den Anderen zu ehren. Wer aber den Dienst empfängt, ehrt den Dienenden nur dann, wenn er auf dessen Ausbeutung verzichtet. Er ehrt den Dienenden, indem er dessen Loyalität als Gabe behandelt. Gott ehrt den Abraham, er erweist ihm Anerkennung dafür, dass er bereit war, sein Liebstes aufs Spiel zu setzen.

\section{Liebesdienste}

Abrahams Dienstbarkeit ist Ausdruck der Freiheit des urteilsmächtigen Mannes, der Ja und Nein sagen kann, und in der Lage ist, sich dem Gottesbezug zu öffnen oder zu verschliessen. Die Bereitschaft, dem zu dienen, an den man sich bindet, ist nicht Unterwerfung und nicht Kritiklosigkeit. Die Selbstdezentrierung zugunsten der Anliegen des Objekts ist nicht blind; das dienende Engagement ist vielmehr Ausdruck dessen, dass der Anspruch des Objekts im Zentrum steht. Man übernimmt die Anliegen des Objekts, beurteilt die Situation in seinem Interesse und mobilisiert die eigenen materiellen und mentalen Ressourcen, um am Objekt guten Dienst - klug, wirksam, auf umsichtige Weise - zu tun. In dieser Weise ist auch 
Abraham herausgefordert, als Sarah will, dass er seinen Sohn Ismail verstösst und dem Tode preisgibt. Abraham nimmt dieses Ansinnen mit grossem Kummer auf, doch kommt es auch hier nicht zum kritischen Disput zwischen den Eheleuten. Ismail wird verstossen, Gott sorgt dafür, dass er und seine Mutter in der Wüste überleben.

Wer in der Liebe dem Anderen dient, engagiert sich für das, was ihn ausmacht, was seine Anliegen sind und erweist ihm damit Ehre ohne Einschränkung. Er verzichtet auf den Vorbehalt, er verzichtet auf Kontrolle. Zugleich gibt er einen mächtigen Vertrauenskredit: Er geht davon aus, dass sein Dienen Anerkennung findet und nicht ausgebeutet wird. Und wenn doch? Dann kommt es, unter Schmerzen, zur Lossagung aus der Bindung. Für dich war ich nur ein nützlicher Idiot, mag einer sagen, der sich von der Geliebten ausgebeutet sieht und sich von ihr lossagt.

\section{Klugheit, List und Dienst am Nächsten}

In dem 1862 erschienenen Roman Die Elenden von Victor Hugo wird nach 19 Jahren Haft der Galeerensträfling Jean Valjean freigelassen. Überall wird der Freigelassene verjagt. Dann aber findet er Aufnahme beim Bischof von Digne, wird sein Gast und nimmt teil am Abendessen. Der Bischof von Digne bewirtet ihn liebenswürdig und bereitet ihm ein Zimmer zum Übernachten. So sehr den ehemaligen Sträfling die Gastfreundschaft des Hausherrn bewegt, er stiehlt trotzdem das Silber aus dem unverschlossenen Schrank und verlässt das Haus als ein Dieb. Die Polizei greift ihn auf und stellt ihn dem Bischof gegenüber. Dieser erklärt, er habe Jean Valjean das Silber geschenkt. So skeptisch die Polizei ist, Jean Valjean bleibt auf freiem Fuss. Die alte Schwester des Bischofs macht ihmVorhaltungen: Einen Diebstahl vertuschen - das geht nicht. Der Bischof aber hält ihr entgegen, er habe keinen Anspruch auf das Silber, das bloss ererbt, nicht erworben sei.

Jean Valjean, der Gast, hatte Teil an den Gütern des Hauses, der Bischof ehrte ihn als Gast: Dienst am Nächsten in kultivierter Heiterkeit. Jean Valjean aber beutet den Dienenden aus. Der Bischof reagiert weder kritisch noch strafend noch zweifelnd, in der Haltung mentaler Reserve. Vielmehr vollendet sich die Praxis des Dienstes. Er beschenkt seinen Gast. Er selbst ist ja - so entgegnet er seiner Schwester - ein Beschenkter, denn das Silber ist ihm, wie er sagt, nur zugefallen, er hatte daran seine Freude, aber keinen Anspruch. Naive Ignoranz ist beim Bischof nicht am Werk, auch nicht sentimentales 
Sympathisieren, vielmehr die leise Stimme des Intellekts im Bund mit Humor und mitmenschlichem Interesse.

\section{Literatur}

Boothe, B. (2006): Abraham, der Ausgezeichnete. Die Loyalitätsprobe. Handlung Kultur Interpretation. Zeitschrift für Sozial- und Kulturwissenschaften 15:274-304.

Boothe, B. (2006a): Die Religionskritik Freuds und die Überschreitung des Gegebenen. In:I.U. Dalferth/H.-P. Grosshans (Hg.). Kritik der Religion, Tübingen: Mohr/Siebeck: 163-186.

Freud, Sigmund (1927): Die Zukunft einer Illusion. Gesammelte Werke XIV, London: Imago 1948, 325-380.

Freud, Sigmund (1928): Der Humor. Gesammelte Werke XIV, London: Imago 1948, 381-390.

Hugo, Victor (2006): Die Elenden, Düsseldorf: Patmos (Ersterscheinen 1862).

Kant, I. (1798): Der Streit der Fakultäten. In.: W. Weischedel (Hg.). Band sechs der Werke in sechs Bänden, Darmstadt: Wissenschaftliche Buchgesellschaft 1975: 333 .

Mackie, J. L. (1987): Das Wunder des Theismus. Argumente für und gegen die Existenz Gottes, Stuttgart: Reclam.

— Prof. Dr. phil. Brigitte Boothe ist Ordinaria für Klinische Psychologie an der Universität Zürich und Leiterin der psychotherapeutischen Praxisstelle in psychoanalytischer Ausrichtung des Psychotherapeutischen Zentrums der Universität Zürich. 